\section{Identification of virulence and antibiotic resistance factors in Arcobacter butzleri isolated from bovine milk by Whole Genome Sequencing}

\author{
Antonio Parisi, ${ }^{1}$ Loredana Capozzi, \\ Angelica Bianco, ${ }^{1}$ Marta Caruso, ${ }^{1}$ \\ Laura Latorre, ${ }^{1}$ Antonella Costa, ${ }^{2}$ \\ Anna Giannico, ${ }^{1}$ Donato Ridolfi, ${ }^{1}$ \\ Carmela Bulzacchelli, ${ }^{1}$ \\ Gianfranco Santagada ${ }^{1}$
}

${ }^{1}$ Experimental Zooprophylactic Institute of Apulia and Basilicata, Foggia;

${ }^{2}$ Experimental Zooprophylactic Institute of Sicily, Palermo, Italy

\begin{abstract}
Arcobacter butzleri is a pathogenic aerobic bacterium responsible for diarrhea and septicemia in humans. It is frequently isolated from food products of animal origin, including milk and dairy products. To data, few reports are currently available on the genetic characteristics and virulence profiles of $A$. butzleri. The aim of this study was to investigate the genetic diversity and to characterize the virulence and antibiotic resistance profiles of 10 A. butzleri strains isolated from bovine milk samples by Whole Genome Sequence (WGS). Multi-Locus Sequence Typing (MLST) revealed that three isolates belonged to the ST66, two to the ST420 and the remaining five strains to the ST627, ST629, ST630, ST633 and ST637, respectively. The $100 \%$ of the strains carried $c a d F$, pldA, ciaB, cj1349, mviN and tly $A$ virulence factors genes; $60 \%$ iroE; $50 \% \operatorname{irg} A ; 10 \%$ hecB Resistome prediction showed a multidrug resistance: $100 \%$ of isolates resulted resistant to fluoroquinolones and tetracycline; $90 \%$ of strains to rifampicin and cephalosporins and a minor percentage to other antibiotics. Furthermore, the $50 \%$ of strains harbored four mutations in Mycobacterium tuberculosis kat $G$ gene conferring resistance to isoniazid. The study provided interesting data on the virulence characteristics and on the genetic endowment related to the antimicrobial resistance of $A$. butzleri isolates from milk. The determination of the STs also added information concerning the genetic variability of this microorganism. To date, a very limited number of studies have been published on the typing of $A$. butzleri using WGS, so this paper proposes an innovative methodological approach that allows a rapid and complete characterization of pathogenic microorganisms.
\end{abstract}

\section{Introduction}

The Arcobacter genus is ubiquitous in animals, in a variety of foods of animal and non-animal origin and in aquatic environments; it has been linked to animal and human illness (Collado and Figueras, 2011). Arcobacter spp. have been isolated from faces of numerous species of farm animals and found to contaminate different foods, including milk. In industrialized countries, the consumption of raw or undercooked food or the ingestion of Arcobacter-contaminated water represent the most important sources of human infection. To date, the lack of highly standardized methods for the isolation of Arcobacter spp. could affect the evaluation of the real prevalence of this potential pathogen in food that is believed to be underestimated (Prouzet-Mauleon et al., 2006). Furthermore, the prevalence of Arcobacter spp. varies from species to species and from country to country, but in general Arcobacter spp. it is principally found in poultry followed from pigs and cattle (van Driessche et al., 2003). Additionally, there are studies that displayed also the presence of Arcobacter spp. in other species of birds (i.e. ducks, turkeys, domestic geese) and in mussels (Collado et al., 2009; Mottola et al., 2016). Few studies investigated the presence of Arcobacter spp. in bovine milk and the prevalence ranged from 1 to $46 \%$ (Collado and Figueras, 2011).

A. butzleri, A. cryaerophilus and A. skirrowii, have been associated with gastrointestinal cases in humans (Collado and Figueras, 2011). A. butzleri is associated with enteritis and diarrhea in pigs, cattle and horses; A. skirrowii is usually associated with diarrhea and hemorrhagic colitis in sheeps and cattle; A. cryaerophilus has been described as cause of abortion in animals, event rarely associated also to $A$. butzleri and A. skirrowii (Collado and Figueras, 2011; Mottola et al., 2016; Ramees et al., 2017). A. butzleri is the species of arcobacters most commonly reported as contaminant of foods (Collado et al., 2009). A. butzleri represents an environmental bacterium that has developed a high level of niche adaptation (Merga et al., 2013) and the ability to survive in the adverse conditions like food processing and storage (Collado and Figueras, 2011; Ferreira et al., 2016). In recent years the increased number of cases of illness has raised the concern that this microorganism is a serious risk to human health (International Commission on Microbial Specification for Foods (ICMSF, 2002). The knowledge of the mechanisms of pathogenicity of $A$. butzleri is still limited (Ferreira and Glassey, 2017). Adhesion,
Correspondence: Antonio Parisi, Istituto Zooprofilattico Sperimentale della Puglia e della Basilicata, via Chiancolla 1, 70017 Putignano (BA), Italy.

Tel.: +39.080.4057858 - Fax: +39.080.4057753. E-mail: antonio.parisi@izspb.it

Key words: Arcobacter butzleri, whole genome sequencing, virulence factor, resistome.

Contributions: the authors contributed equally. Conflict of interest: the authors declare no potential conflict of interests.

Funding: Fondi Ricerca Corrente Ministero della Salute IZS PB 06/14 RC.

Received for publication: 19 September 2018. Revision received: 4 December 2018.

Accepted for publication: 4 December 2018

This work is licensed under a Creative Commons Attribution-NonCommercial 4.0 International License (CC BY-NC 4.0).

(C) Copyright A. Parisi, et al., 2019

Licensee PAGEPress, Italy

Italian Journal of Food Safety 2019; 8:7840

doi:10.4081/ijfs.2019.7840

invasion and cytotoxicity have been previously examined in vitro using different cell lines (Karadas et al., 2013). Very little information is known about the genes involved in the virulence mechanisms (Collado and Figueras, 2011). It was found that this species possesses presumed virulent determinants such as the $c a d F$ and $c j 1349$ genes, encoding fibronectin-binding proteins, $c i a B$ that encodes a Campylobacter invasion antigen, the virulence factor $m v i N$ encoding an essential protein required for the biosynthesis of peptidoglycan, pldA that encode phospholipase A, the hemolysin gene tly $A$, the $\operatorname{irg} A$ gene that encodes an iron-regulated outer membrane protein, Iro $E$ gene codes for a periplasmic enzyme functional for iron acquisition and required to establish and maintain infection, hecA, a member of the filamentous hemagglutinin (FHA) family, and $h e c B$ that encodes a hemolysin activation protein (Miller et al., 2007).

Currently, numerous genotyping methods are used for epidemiological molecular studies and each of them shows a different discriminatory power (Giacometti et al., 2018). Among them, MLST presents the advantage of the availability of an online database for Arcobacter spp. (https://pubmlst.org/arcobacter/) that allows to compare strains isolated from different studies and from various geographical areas (De Cesare et al., 2016; Merga et al., 2013). Whole Genome Sequencing (WGS) of 
microbial strains is becoming, due to the continuous decline of the associated costs, a powerful, very affordable and fast tool for microbial typing, allowing a much higher resolution than traditional typing methods, and an important tool for public health surveillance and outbreak investigation. Recent studies have shown that WGS analysis can potentially be a single, rapid and cost-effective approach to define resistance genotypes and predict resistance phenotypes of bacteria with great sensitivity and specificity (McDermott et al., 2016)

The aim of the present study was to assess the genetic characteristics of 10 strains of $A$. butzleri isolated in raw bovine milk samples collected in Apulia Region by WGS followed by in silico prediction of the virulence factors and the resistome.

\section{Materials and Methods}

\section{Strain collection and identification}

Bulk tank milk samples were collected from 190 dairy farms of Apulia Region during the year 2017. Milk samples (10 mL) were homogenized with $90 \mathrm{~mL}$ of Arcobacter Enrichment Broth plus Cefoperazone, Amphotericin B, Teicoplanin (CAT) Selective Supplement and incubated at $30^{\circ} \mathrm{C}$ for $48 \mathrm{~h}$ in microaerophilic conditions. From each broth DNA was extracted and subjected to an Arcobacter genus - specific Real Time PCR as described elsewhere (Gonzalez et al., 2014). The PCR positive broths were filtered using $0.45 \mu \mathrm{m}$ pore size syringe filters and $0.2 \mathrm{~mL}$ were spread onto plates of Columbia Blood Agar, MCCD Agar and Karmali Agar. The plates were incubated at $30^{\circ} \mathrm{C}$ for 3-4 days in microaerophilic conditions and checked daily. Presumptive identification tests (Gram staining, catalase and oxidase tests) were performed on at least 5 suspected colonies among those grown on Columbia Blood Agar, MCCD Agar and Karmali Agar. Colonies of Gram-negative spiral bacteria, oxidase and catalase positive were considered presumptive Arcobacter spp. and subjected (one colony for each sample) to species identification using an atp $A$ amplification and sequencing method (Miller et al., 2014).

\section{WGS}

Ten isolates, all identified as A. butzleri, were characterized by WGS. Genomic DNA was extracted from the isolates using DNeasy Blood \& Tissue Kit (Qiagen, Hilden, Germany), according to manufacturer's protocol. An indexed genomic library for each isolate was prepared using the Nextera XT DNA sample preparation kit (Illumina, San Diego, CA), according to manufacturer's instruction. Sequencing was performed on MiSeq platform (Illumina) using the MiSeq Reagent kit v2, 2x250 paired-end cycles (Illumina). The reads obtained for each sample were trimmed, subjected to quality filtering using Trimmomatic (Bolger et al., 2014), and finally assembled by SPAdes v 3.11 (Nurk et al., 2013). General features of the A. butzleri genome sequences were collected in Table 1 . For each genome, a script for the extraction of the sequences of the MLST alleles was used in Bionumerics 7.6 (Applied Maths, Belgium). Allele profiles and Sequence Types (STs) were assigned according to Arcobacter MLST database (https://pubmlst.org/arcobacter/). Assembled genomes were submitted to Virulence Factors Database (VFDB: http://www. mgc.ac.cn/VFs/) for the detection of virulence factors (VFs). For virulence gene identification, BLASTn method against VDFB was used considering only the genes with E Value equal to 0. Additionally, BLAST in silico analysis was performed for typical virulence factors of $A$. butzleri (i.e. cadF, cj1349, ciaB, pldA, tlyA, hecA, hecB, $m v i N, \operatorname{irg} A$, IroE).

In order to evaluate the presence of antimicrobial resistance (AMR) genes, assembled genomes were submitted to Resistance Gene Identifier (RGI) software

Table 1. General features of the Arcobacter butzleri genome sequences.

\begin{tabular}{lccccccccccc} 
& AB141 & AB142 & AB143 & AB144 & AB147 & AB149 & AB151 & AB155 & AB157 & AB158 \\
Deep coverage & $50 X$ & $40 X$ & $50 X$ & $60 X$ & $40 X$ & $35 X$ & $35 X$ & $60 X$ & $40 X$ & $35 X$ \\
contigs & 107 & 140 & 103 & 85 & 145 & 179 & 195 & 61 & 125 & 167 \\
\hline Largest contig & 97506 & 96476 & 99956 & 188708 & 76532 & 78825 & 65537 & 226837 & 95595 & 87642 \\
Total length & 2296956 & 2279850 & 2389007 & 2395935 & 2119859 & 2392732 & 2470188 & 2359823 & 2268926 & 2340572 \\
\hline N50 & 36402 & 24517 & 35185 & 52313 & 23090 & 24168 & 21682 & 76682 & 32179 & 22046 \\
N75 & 17051 & 15513 & 24226 & 24074 & 12615 & 11360 & 11399 & 48341 & 15033 & 13242 \\
\hline L50 & 20 & 27 & 21 & 14 & 30 & 30 & 33 & 9 & 22 & 33 \\
L75 & 43 & 57 & 41 & 31 & 61 & 67 & 74 & 19 & 50 & 67 \\
\hline GC (\%) & 27.12 & 27.17 & 26.7 & 26.85 & 27.1 & 27.02 & 26.99 & 26.91 & 27.06 & 27.11 \\
\hline
\end{tabular}

Table 2. Diversity of A. butzleri. Alleles and sequence types (ST).

\begin{tabular}{|c|c|c|c|c|c|c|c|c|}
\hline ID Strain & aspA & $\operatorname{atpA}$ & $g \ln A$ & gltA & $g l y A$ & $p g m$ & $t k t$ & ST \\
\hline AB141 & 15 & 10 & 1 & 17 & 19 & 2 & 13 & 66 \\
\hline AB142 & 15 & 10 & 1 & 17 & 19 & 2 & 13 & 66 \\
\hline $\mathrm{AB} 143$ & 6 & 23 & 1 & 11 & 494 & 58 & 199 & 627 \\
\hline $\mathrm{AB} 144$ & 48 & 25 & 41 & 19 & 487 & 101 & 272 & 633 \\
\hline $\mathrm{AB} 147$ & 5 & 5 & 9 & 15 & 120 & 7 & 6 & 629 \\
\hline AB149 & 23 & 17 & 17 & 19 & $461 / 176$ & 11 & 65 & 630 \\
\hline $\mathrm{AB} 151$ & 5 & 5 & 5 & 15 & $176 / 66$ & 11 & 10 & 420 \\
\hline $\mathrm{AB} 155$ & 310 & 133 & 11 & 19 & $192 / 19$ & 123 & 271 & 637 \\
\hline $\mathrm{AB} 157$ & 15 & 10 & 1 & 17 & 19 & 2 & 13 & 66 \\
\hline AB158 & 5 & 5 & 5 & 15 & $176 / 66$ & 11 & 10 & 420 \\
\hline
\end{tabular}


of Comprehensive Antibiotic Resistance Database (CARD) (https://card.mcmaster. $\mathrm{ca} /$ ), considering the genes with $50 \%$ identity of matching region.

\section{Results}

The Arcobacter genus - specific PCR revealed the presence of Arcobacter spp. in 25/190 (13\%) milk samples analyzed. The following microbiological procedures allowed to isolate suspected colonies just in 10 of the 25 (40\%) PCR-positive samples. Presumptive identification tests performed on suspected colonies always identified Gram-negative spiral bacteria, oxidase and catalase positive. The presumptive Arcobacter spp. colonies were identified as A.butzleri using the partial sequencing of the atp $A$ gene. Prevalence of Arcobacter spp. in bulk tank milk was 5\%(10/190).

MLST genes revealed a high genetic diversity in A. butzleri isolates. The number of alleles per locus observed in the isolates were showed in Table 2. Seven different STs were identified: $30 \%(3 / 10)$ of strains belonged to ST66 and 20\% (2/10) to ST420. One single isolate was identified as the following STs: ST627, ST629, ST630, ST633, ST637, respectively. Interestingly, the $40 \%(4 / 10)$ of strains showed two alleles for glyA genes (Table 2), previously identified as glyAl and glyA2 (Miller et al., 2009). Submission of genome sequences in VFDB allowed to identify more of 400 virulence factors (data not shown). Among them only 12 showed a perfect identity (Evalue $=0.00$ ) with similar virulence factors of Campylobacter spp. Interestingly, only two of them ( $c a d F$ and $c i a B$ ) have been described as virulence factors in A. butzleri. A further search of the determinants known to play a role in $A$. butzleri infections was performed using BLASTn with the aim to detect 10 specific virulence factors. This approach allowed to find $90 \%(9 / 10)$ (Table 3 ) of the virulence factors with the following distribution: the $100 \%(10 / 10)$ of them carried $c a d F, c i a B, c j 1349, m v i N$, pldA and tly $A$ genes; the $60 \%$ of them carried the iro $E$ gene; the $50 \%$ of them carried $\operatorname{irg} A$ gene; the $10 \%$ of them carried $h e c B$ gene. Any of them isolates carried hecA gene (Table 3).

The prediction of resistome by CARD showed that the $100 \%$ of strains harbored the adeF gene, that confers resistance to fluoroquinolone and tetracycline antibiotics; the $90 \%$ of them harbored $a c r B$ gene that confers resistance to rifamycin, cephalosporin, triclosan, glycylcycline, tetracycline, penam, phenicol and fluoroquinolone antibiotics; the $30 \%$ of strains harbored pmrE gene that confers resistance to peptide antibiotic; the $10 \%$ of strains carried $a a d A 2$ and $m a c B$ genes, that confers the resistance to aminoglycoside antibiotic and macrolide antibiotic, respectively. Furthermore, the $50 \%$ of strains harbored mutations (S140N, A139V, R463L, A379T) in Mycobacterium tuberculosis kat G gene conferring resistance to isoniazid (Table 4).

\section{Discussion}

Arcobacter are considered to be an emergent food pathogen, but a reduced number of studies is available and little is known about the pathogenic mechanism (Collado and Figueras, 2011). To date a total of 26 recognized Arcobacter species have been described (Vicente-Martinsa et al., 2017); among these, A. butzleri, $A$. cryaerophilus and $A$. skirrowii have been shown to cause infection in humans. The major transmission pathway of these bacteria is represented by the ingestion contaminated

Table 3. Presence of main virulence genes in A.butzleri isolates from milk.

\begin{tabular}{|c|c|c|c|c|c|c|c|c|c|c|}
\hline ID Strain & & & & & ence $f$ & tors & & & & \\
\hline & $c a d F$ & $c i a B$ & cj1349 & $\operatorname{irg} A$ & hecA & hecB & moiN & pldA & $t l y A$ & $\operatorname{iro} E$ \\
\hline AB141 & + & + & + & - & - & - & + & + & + & - \\
\hline AB142 & + & + & + & - & - & - & + & + & + & - \\
\hline AB143 & + & + & + & - & - & - & + & + & + & - \\
\hline AB144 & + & + & + & + & - & - & + & + & + & + \\
\hline $\mathrm{AB} 147$ & + & + & + & + & - & - & + & + & + & + \\
\hline AB149 & + & + & + & + & - & - & + & + & + & + \\
\hline AB151 & + & + & + & + & - & - & + & + & + & + \\
\hline AB155 & + & + & + & - & - & + & + & + & + & - \\
\hline $\mathrm{AB} 157$ & + & + & + & - & - & - & + & + & + & + \\
\hline AB158 & + & + & + & + & - & - & + & + & + & + \\
\hline
\end{tabular}

+ , positive; - negative.

Table 4. Presence of AMR genes in A. butzleri isolates from milk.

\begin{tabular}{lcccccc} 
ID Strain & & \multicolumn{5}{c}{ AMR genes } \\
& adeF & aadA2 & acrB & PmrE & macB & katG mut \\
$\mathrm{AB} 141$ & + & - & + & - & - & - \\
$\mathrm{AB} 142$ & + & - & + & - & - & - \\
\hline $\mathrm{AB} 143$ & + & - & + & - & - & - \\
$\mathrm{AB} 144$ & + & - & + & + & - & - \\
\hline $\mathrm{AB} 147$ & + & - & + & - & + & + \\
$\mathrm{AB} 149$ & + & + & - & - & - & + \\
$\mathrm{AB} 151$ & + & - & + & + & - & + \\
$\mathrm{AB} 155$ & + & - & + & - & - & + \\
$\mathrm{AB} 157$ & + & - & + & - & - & - \\
$\mathrm{AB} 158$ & + & - & + & + & - & + \\
\hline
\end{tabular}

water or food, particularly raw or minimally processed foods are generally considered the main source of human infection in industrialized countries (Van Driessche and Houf, 2008). Prevalence of Arcobacter spp. in analyzed milk samples was $5 \%$, although 25 samples (13\%) resulted PCR positive. Despite the little number of studies that investigated the presence of Arcobacter spp. in bovine milk, there are evidence showing a prevalence rates of $5.8 \%, 15 \%$ and $46 \%$ in Malaysia, Finland and Northern Ireland, respectively; in Italy a prevalence rate of $26 \%(25 / 190)$ was found in bulk tank milk produced in Lombardia Region (Collado and Figueras, 2011). Although the different prevalence rates reported in literature could be due to different sampling methods and to the absence of a standardized protocol of analysis. The entire draft genome of isolates was used to predict the STs and this analysis highlight a considerable amount of genetic diversity between the $A$. butzleri isolates (De Cesare et al., 2016). That result was consolidated by the identification of 5 STs (ST627; ST629; ST630; ST633; ST637) that 
were previously unreported. Interestingly, these STs were represented by a single isolate. More than one isolate was identified as ST66 and ST420, both of whom were previously described as food contaminants: ST66 in ricotta cheese (De Cesare et al., 2016; Giacometti et al., 2018) and ST420 in raw WB milk, bulk tank valve, milk pump and mozzarella cheese molding roller (De Cesare et al., 2016; Giacometti et al., 2018). As more than 600 profiles are currently available in the PubMLST database, the high number of STs identified clearly shows the high genetic diversity of $A$. butzleri as previously described (De Cesare et al., 2016; Miller et al., 2009). The high variability of A. butzleri would be due to the genetic recombination causing rapid genomic changes (De Cesare et al., 2016). Interestingly, ST420 has been also identified in strains isolated in nonfood contact surfaces (De Cesare et al., 2016; Giacometti et al., 2018). Taken together, these finding suggest that the detection of very different occurrence values in studies performed on the same sample type and using similar protocols could be related to numerous factors such as hygiene of farms, source of water, feeding, climate etc (Collado and Figueras, 2011). A. butzleri was the only isolated species in the analyzed bulk tank milk samples, in agreement with the results of previous studies that reported it as the most commonly recovered species both in milk, milk products, and in dairy plants (Serraino and Giacometti, 2014). In previous studies it was reported that $A$. butzleri showed the ability to grow in several substrates and in different environmental conditions and to survive to sanitizing procedures, characteristics that make the bacterium adapt to resist in the environment and to cause secondary food infections (Serraino and Giacometti, 2014). For this reason, it become very important to investigate the presence of virulenceassociated genes and antibiotic resistance genes. In our study the most commonly detected genes were: $c a d F$, that encodes a protein able to induces the internalization of bacterial cells by the activation of GTPases; $c i a B$, that encodes an invasion protein injected directly into the cytoplasm of the host cells by a secretion system; $c j 1349$, that encodes an adhesion protein that binding specifically to fibronectin; $m v i N$, that encodes an inner membrane protein required for peptidoglycan biosynthesis; pldA, that encodes outer membrane phospholipase A associated with lysis of erythrocites; tly $A$, that encodes a hemolysin. This result was in according to previous data (Girbau et al., 2015; Mottola et al., 2016) that investigated A. butzleri isolated from different hosts and environments like food products, processing water, fecal samples etc.

The periplasmic enzyme gene iroE, which was found in $60 \%$ of isolates, and the iron-regulated outer-membrane gene $\operatorname{irg} A$, that was found in $50 \%$ of isolates, are functional components for the iron acquisition in E. coli and therefore required for establishing and maintaining infections (Rashid et al., 2006; Zhu et al., 2005). Finally, the hemolysin activation gene hecB was found in a strain. The low prevalence of them were in agreement with previous studies (Ferreira et al., 2014; Girbau et al., 2015; Mottola et al., 2016). Any of our isolates carried hecA gene which encodes a hemagglutinin in uropathogenic $E$. coli. This result was in accord to a previous study (Gibau et al., 2015), but it might be due to the lower number of isolates included in our study. All these genes are implicated in adhesion and invasion mechanisms that are abilities necessary for colonization and infection. These characteristics confirmed the pathogenic potential of A. butzleri isolates from milk. Despite the small number of analyzed isolates, the presence of these genes in the majority of $A$. butzleri isolates in our and in previous studies suggests that they represent competitive factors for the maintenance of $A$. butzleri circulation in the different host populations and consequently in different environmental sites (Piva et al., 2017). Although in vitro cell culture assays have been performed to investigate the role of these virulence genes in adhesion, invasion and cytotoxicity, to date no correlations have been found (Karada et al., 2013; Vandenberg et al., 2014).

The increasing occurrence of $A$. butzleri in foods as well as the ability of these bacteria to resist in different environmental conditions and to cause human disease, put the focus on antimicrobial resistance. $A$. butzleri presents resistance to several classes of antimicrobial agents, being considered as having multidrug resistance (Ferreira et al., 2016). In our study all isolates were predicted to resist to fluoroquinolone and tetracycline antibiotics, whereas $90 \%$ of isolates had determinants linked to the resistance to rifamycin, cephalosporin, triclosan, glycylcycline, penam, phenicol antibiotics. Only a small percentage of all isolates possessed genes responsible of resistance to peptide antibiotics $(30 \%)$, aminoglycosides (10\%) and macrolides $(10 \%)$. Furthermore, we found, in $50 \%$ of isolates, mutations in $k a t G$ that catalyze the activation of isoniazid. Isoniazid inhibits mycolic acid synthesis, which prevents cell wall synthesis in Mycobacteria. Mutations in katG result in inability to activate isoniazid (Tseng et al., 2015). Taken together, these findings highlight the multidrug resistance of $A$. butzleri strains. In previous studies cephalosporin resistance were commonly found in A. butzleri; as matter of fact, cephalosporins are added into the Arcobacter selective media to suppress the growth of accompanying microorganisms (Atabay and Aydin, 2001). Moreover, it has been reported a human case of infection by $A$. butzleri, which resulted resistant to chloramphenicol and ampicillin (Villalobos et al., 2013). Interestingly, the majority of studies are agreeing to report that A. butzleri isolates are highly susceptible to tetracycline, so that it can be used for treatment of human Arcobacter infections (Ramees et al., 2017). Based on our results, that showed a wide occurrence in the $A$. butzleri genomes of genetic determinants potentially involved in Tetracycline resistance, the sensitivity towards this class of antimicrobials should be carefully monitored to avoid therapeutic failure. These results should be confirmed by in vitro phenotypical assays in order to follow the evolution of resistance in this species and to allow the better choice of antimicrobials to be used in clinical therapy. Moreover, other studies are needed to understand the molecular mechanisms of antimicrobial resistance in A. butzleri.

According to literature, our results confirmed the importance of raw milk as a possible source of Arcobacter spp. infection for humans. Consumption of heat-treated milk and the use of pasteurized milk for the production of dairy products should be an effective measure to prevent the risk to human health. However, it is necessary to consider the possible risks on human health consequent to the widespread consumption of dairy products based on raw milk, especially considering that in some of them, such as mozzarella and ricotta cheese, it has been shown that Arcobacter spp. is able to withstand the temperatures used during production (Giacometti et al., 2014).

\section{Conclusions}

In the present study, ten $A$. butzleri strains were analyzed using the WGS approach, which rapidly allowed to define the ST, reveling five new STs, to identify the presence of nine of most representative putative virulence genes and several antibiotic resistance genes. Our results confirmed the usefulness of WGS to obtain relevant information about this important food-borne pathogen in detecting virulence and antimicrobial resistant genes or for genotyping.

The availability of high-performing 
online databases to get prediction analysis, based on genome sequences, will ensure to obtain ever more information in the risk assessment connected to microbial pathogens making this approach particularly suitable for genetic background analysis and to perform epidemiological investigations.

\section{References}

Atabay HI, Aydin F., 2001. Susceptibility of Arcobacter butzleri isolates to 23 antimicrobial agents. Lett Appl Microbiol 33:430-3.

Bolger AM, Lohse M, Usadel B, 2014. Trimmomatic: a flexible trimmer for Illumina sequence data. Bioinformatics 30:2114-20.

Collado L, Figueras MJ, 2011. Taxonomy, epidemiology, and clinical relevance of the genus Arcobacter. Clin Microbiol Rev 24 174-92.

Collado L, Guarro J, Figueras MJ, 2009. Prevalence of Arcobacter in meat and shellfish. J Food Prot 72:1102-6.

De Cesare A, Parisi A, Giacometti F, Serraino A, Piva S, Caruso M, De Santis EP, Manfreda G, 2016. Multilocus sequence typing of Arcobacter butzleri isolates collected from dairy plants and their products, and comparison with their PFGE types. J Appl Microbiol 120:165-74.

Ferreira GNM, Glassey J, 2017. Editorial: The European Symposium on Biochemical Engineering Sciences, Dublin 2016. Biotechnol J 12.

Ferreira S, Queiroz JA, Oleastro M, Domingues FC, 2014. Genotypic and phenotypic features of Arcobacter butzleri pathogenicity. Microb Pathog 76:19-25.

Giacometti F, Piva S, Vranckx K, De Bruyne K, Drigo I, Lucchi A, Manfreda G, Serraino A, 2018. Application of MALDI-TOF MS for the subtyping of Arcobacter butzleri strains and comparison with their MLST and PFGE types. Int J Food Microbiol 277:50-7.

Giacometti F, Serraino A, Pasquali F, De Cesare A, Bonerba E, Rosmini R, 2014. Behavior of Arcobacter butzleri and Arcobacter cryaerophilus in ultrahightemperature, pasteurized, and raw cow's milk under different temperature conditions. Foodborne Pathog Dis 11:15-20.

Girbau C, Guerra C, MartinezMalaxetxebarria I, Alonso R, Fernandez-Astorga A, 2015. Prevalence of ten putative virulence genes in the emerging foodborne pathogen
Arcobacter isolated from food products. Food Microbiol 52:146-9.

Gonzalez I, Fernandez-Tome S, Garcia T, Martin R, 2014. Genus-specific PCR assay for screening Arcobacter spp. in chicken meat. J Sci Food Agric 94:1218-24.

Karadas G, Sharbati S, Hanel I, Messelhausser U, Glocker E, Alter T, Golz G, 2013. Presence of virulence genes, adhesion and invasion of Arcobacter butzleri. J Appl Microbiol 115:583-90.

McDermott PF, Tyson GH, Kabera C, Chen Y, Li C, Folster JP, Ayers SL, Lam C, Tate HP, Zhao S, 2016. Whole-Genome Sequencing for Detecting Antimicrobial Resistance in Nontyphoidal Salmonella. Antimicrob Agents Chemother 60:5515-20.

Merga JY, Williams NJ, Miller WG, Leatherbarrow AJ, Bennett M, Hall N, Ashelford KE, Winstanley C, 2013. Exploring the diversity of Arcobacter butzleri from cattle in the UK using MLST and whole genome sequencing. PLoS One 8:e55240.

Miller WG, Parker CT, Rubenfield M, Mendz GL, Wosten MM, Ussery DW, Stolz JF, Binnewies TT, Hallin PF, Wang G, Malek JA, Rogosin A, Stanker LH, Mandrell RE, 2007. The complete genome sequence and analysis of the epsilonproteobacterium Arcobacter butzleri. PLoS One 2:e1358.

Miller WG, Wesley IV, On SL, Houf K, Megraud F, Wang G, Yee E, Srijan A, Mason CJ, 2009. First multi-locus sequence typing scheme for Arcobacter spp. BMC Microbiol 9:196.

Miller WG, Yee E, Jolley KA, Chapman $\mathrm{MH}, 2014$. Use of an improved atpA amplification and sequencing method to identify members of the Campylobacteraceae and Helicobacteraceae. Lett Appl Microbiol 58:582-90.

Mottola A, Bonerba E, Figueras MJ, PerezCataluna A, Marchetti P, Serraino A, Bozzo G, Terio V, Tantillo G, Di Pinto A., 2016. Occurrence of potentially pathogenic arcobacters in shellfish. Food Microbiol 57:23-27.

Nurk S, Bankevich A, Antipov D, Gurevich AA, Korobeynikov A, Lapidus A, Prjibelski AD, Pyshkin A, Sirotkin A, Sirotkin Y, Stepanauskas R, Clingenpeel SR, Woyke T, McLean JS, Lasken R, Tesler G, Alekseyev MA, Pevzner PA, 2013. Assembling singlecell genomes and mini-metagenomes from chimeric MDA products. J Comput Biol 20:714-7.
Piva S, Gariano GR, Bonilauri P, Giacometti F, Decastelli L, Florio D, Massella E, Serraino A, 2017. Occurrence of putative virulence genes on Arcobacter butzleri isolated from three different environmental sites throughout the dairy chain. J Appl Microbiol 122:1071-7.

Prouzet-Mauleon V, Labadi L, Bouges N, Menard A, Megraud F, 2006. Arcobacter butzleri: underestimated enteropathogen. Emerg Infect Dis 12:307-9.

Ramees TP, Dhama K, Karthik K, Rathore RS, Kumar A, Saminathan M, Tiwari R, Malik YS, Singh RK, 2017. Arcobacter: an emerging food-borne zoonotic pathogen, its public health concerns and advances in diagnosis and control - a comprehensive review. Vet Q 37:13661.

Serraino A, Giacometti F, 2014. Short communication: occurrence of Arcobacter species in industrial dairy plants. J Dairy Sci 97:2061-5.

Tseng ST, Tai CH, Li CR, Lin CF, Shi ZY, 2015. The mutations of katG and inhA genes of isoniazid-resistant Mycobacterium tuberculosis isolates in Taiwan. J Microbiol Immunol Infect 48:249-55

van Driessche E, Houf K, 2008. Survival capacity in water of Arcobacter species under different temperature conditions. J Appl Microbiol 105:443-51.

van Driessche E, Houf K, van Hoof J, De Zutter L, Vandamme P, 2003. Isolation of Arcobacter species from animal feces. FEMS Microbiol Lett 229:243-8.

Vandenberg O, Dediste A, Houf K, Ibekwem S, Souayah H, Cadranel S, Douat N, Zissis G, Butzler JP, Vandamme P, 2004. Arcobacter species in humans. Emerg Infect Dis 10:1863-7.

Villalobos EG, Jaramillo HF, Ulate CC, Echandi ML, 2013. Isolation and identification of zoonotic species of genus arcobacter from chicken viscera obtained from retail distributors of the metropolitan area of San Jose, Costa Rica. J Food Prot 76:879-82.

Vicente-Martinsa S, Oleastrob M, Dominguesa FC, Ferreira S, 2017. Arcobacter spp. at retail food from Portugal: Prevalence, genotyping and antibiotics resistance. Food Control 85:107-12

Zhu M, Valdebenito M, Winkelmann G and Hantke K., 2005. Functions of the siderophore esterases IroD and IroE in iron-salmochelin utilization. Microbiology 151:2363-72. 